\title{
Bit Error Probability of M-QAM Under Impulsive Noise and Fading Modeled by Using Markov Chains
}

\author{
Hugerles S. SILVA ${ }^{1}$, Marcelo S. de ALENCAR ${ }^{2}$, Wamberto J. L. de QUEIROZ ${ }^{1}$, \\ Rodrigo de A. COELHO ${ }^{1}$, Francisco MADEIRO ${ }^{3}$ \\ ${ }^{1}$ Dept. of Electrical Engineering, Federal University of Campina Grande, Campina Grande, Paraíba, Brazil \\ ${ }^{2}$ Dept. of Electrical Engineering, Federal University of Bahia, Salvador, Bahia, Brazil \\ ${ }^{3}$ University of Pernambuco, Recife, Pernambuco, Brazil
}

\{hugerles.silva, rodrigo.almeida\}@ee.ufcg.edu.br, \{wamberto, malencar\}@ dee.ufcg.edu.br, madeiro@ poli.br

Submitted February 13, 2018 / Accepted August 12, 2018

\begin{abstract}
This article presents new exact expressions, written in terms of elementary transcendental functions, for calculating the bit error probability of $M$-ary Quadrature Amplitude Modulation (M-QAM) scheme considering the wireless communication channel modeled by a Markov chain with $N$ states. For the numerical evaluation of the expressions obtained, a particular case of a Markov chain with two states is considered, with each state representing distinct scenarios. In the first scenario it is considered the presence of Gated Additive White Gaussian Noise (GAWGN) and fading $\eta$ - $\mu$ or $\kappa-\mu$, while the second scenario considers the presence of the Double Gated Additive White Gaussian Noise $\left(G^{2} A W G N\right)$ and fading $\eta$ - $\mu$ or $\kappa-\mu$. Bit error probability curves as a function of the signal-to-permanent-noise ratio for different values of the signal-to-impulsive-noise ratio, fading parameters and modulation order $M$ are also presented.
\end{abstract}

\section{Keywords}

Markov chains, $\eta-\mu$ fading, $\kappa-\mu$ fading, bit error probability, impulsive noise

\section{Introduction}

The signal transmitted in industrial environments and indoor environments, such as shopping malls, is subjected to noise from multiple sources and interference in the spectrum band intended for industrial applications in $2.4 \mathrm{GHz}$ [1]. Beyond the noise usually modeled as an additive white Gaussian process, these environments also present noise of impulsive nature that arise mainly due to the presence of numerous thyristorized equipment whose switching can generate disturbances in the wireless links. Electric motors, frequency inverters, welding equipment, triggering of lamps, among others, are examples of sources of impulsive noise [2].
Another challenge commonly encountered in industrial environments is fading, which imposes random variations of intensity on the transmitted signal [1]. This phenomenon arises due to the large number of objects and machines present in these environments, which are usually constructed with metallic materials and provide appropriate conditions to multipath propagation [3]. The combination of phenomena such as impulsive noise and fading can seriously compromise the quality of the communication link.

In such wireless environments, the effects of fading and impulsive noise on the observed signal at the output of a matched filter in the receiver may be modeled by a twoterm composition, one representing the information signal affected by fading and the other term representing the impulsive noise [2].

In non selective frequency channel models, fading is usually considered flat and characterized by a single probability distribution over at least one symbol time [4]. However, in environments such as shopping malls and factories, a single probability distribution may not be able to appropriately model the intensity variations imposed on the transmitted signal as the receiver moves and may experience different environment conditions for multipath propagation. In [2] industrial environments are considered and more than one probability distribution is used to characterize the effects of fading.

A possible way to model sudden changes in the statistical behavior of the transmitted signal intensity, as the receiver moves through the communication environment, is by means of Markov chains [5-7]. One advantage of considering Markov chains, compared to the non selective frequency model, is that they allow to better describe the statistical changes of fading over time [8]. These variations can be caused by changes in the scenarios in which the transmissions are performed and can affect both up and downlinks on reduced-diameter cells as well as horizontal device-to-device links [9]. 
Other authors have also used Markov chains to describe statistical behavior change in the channel, such as Liu et al. [10] and Altinel and Kurt [11]. Lutz et al. [12], for example, defined a channel model with two states, one classified as good and the other classified as bad, depending on the conditions and level of attenuation in each scenario. Another important work is that of Vucetic and Du [13], which characterized the wireless channel of a particular geographical area of Australia by a Markov chain of four states, combining four different types of conditions.

In this work, exact expressions for the computation of the average bit error probability (BEP), $P_{e}$, of $M$-ary Quadrature Amplitude Modulation ( $M$-QAM) scheme are determined considering that the alternations in the fading statistics along the channel are described by the state transitions of a Markov chain with $N$ states. The expressions obtained are novel and written in terms of elementary transcendental functions. For the numerical evaluation of the expressions obtained, a particular case of a Markov chain with two states is considered, with each state representing distinct scenarios. In the first state of the chain, the presence of the Gated Additive White Gaussian Noise (GAWGN) and fading $\eta-\mu$ or $\kappa-\mu$ is considered, whereas in the second state the presence of the Double Gated Additive White Gaussian Noise ( $\left.\mathrm{G}^{2} \mathrm{AWGN}\right)$ and fading $\eta$ - $\mu$ or $\kappa-\mu$ is considered. In the mathematical characterization of the impulsive noise, we have considered the models GAWGN and $\mathrm{G}^{2} \mathrm{AWGN}$ because they are able to characterize, respectively, the occurrence of noisy pulses and bursts of noisy pulses. By its turn, the fading is characterized by the distributions $\eta-\mu$ and $\kappa-\mu$, which are generalist models of fading and encompass, as special cases, the distributions of Rayleigh, Rice and Nakagami, among others, and may be used to characterize fading on a small and large scale, with or without line-of-sight [14].

The methodology used to determine $P_{e}$ is to weight the BEP of the scheme $M$-QAM under the composite noise models GAWGN and $\mathrm{G}^{2} \mathrm{AWGN}$ by the probability density function (PDF) of the fading envelope. In this approach, the representation of Craig [15] is used for the function $\mathrm{Q}(\cdot)$, expressing $P_{e}$ for an integral defined in the range of 0 to $\pi / 2$, in terms of the Moment Generating Function (MGF) of the distributions $\eta-\mu$ and $\kappa-\mu$.

\section{Noise Model}

The mathematical model of noise $\eta(t)$ is given by [16]

$$
\eta(t)=\eta_{\mathrm{g}}(t)+C(t) \eta_{i}(t)
$$

in which $\eta_{i}(t)$ represents a zero-mean complex white Gaussian random process with variance $\sigma_{i}^{2}, C(t)$ is a signal which models the occurrence of noise $\eta_{i}(t)$, characterized by a continuous time and discrete Bernoulli random process, and $\eta_{\mathrm{g}}(t)$ is the background Gaussian noise with zero-mean and variance $\sigma_{\mathrm{g}}^{2}$. The product $C(t) \eta_{i}(t)$, referred to as impulsive noise, in (1), characterizes the noise $\eta_{i}(t)$ gated by the process $C(t)$. The noise can be simple or double gated.
In simple gated noise, the noise is referred to as Gated Additive White Gaussian Noise (GAWGN) and the amplitude of $C(t)$ assumes values zero or one randomly, with probabilities $1-p$ and $p$, respectively. The signal $C(t)$ has unit amplitude in the interval $-\alpha_{p} T / 2 \leq t \leq \alpha_{p} T / 2$ and zero otherwise. The variable $\alpha_{p}$ can assume values between zero and one.

In double gated noise, the noise is referred to as Double Gated Additive White Gaussian Noise ( $\left.\mathrm{G}^{2} \mathrm{AWGN}\right)$ and the random signal $C(t)$ is characterized by the product of two auxiliary processes, $C_{1}(t)$ and $C_{2}(t)$, that take values in the discrete set $\{0,1\}$. In the intervals at which $C_{1}(t)$ assumes value one, several noisy pulses, modeled as $C_{2}(t) \eta_{i}(t)$, can occur. One can say that the interval for which $C_{1}(t)=1$ is a burst of noisy pulses $C_{2}(t) \eta_{i}(t)$. The product $C_{1}(t) C_{2}(t)$ models the occurrence of bursts of noisy pulses. The function $C_{1}(t)$ assumes the values one and zero with probabilities $p_{1}$ and $1-p_{1}$, respectively. This function has unit amplitude in the interval $0 \leq t \leq \beta T_{1}$ and zero otherwise. The signal $C_{2}(t)$ assumes the values one and zero randomly, with probabilities $p_{2}$ and $1-p_{2}$, respectively, and has unit amplitude in the interval $0 \leq t \leq \alpha_{p} T_{2}$ and zero otherwise. The variables $\beta$ e $\alpha_{p}$ assume values between zero and one.

\section{Average Bit Error Probability of $M$-QAM Under Noise and Fad- ing Modeled by Using a $N$ States Markov Chain}

It was shown in [17] that the BEP of $M$-QAM for a given fading $z$, denoted by $P(e \mid z)$, under GAWGN, can be written as

$$
\begin{aligned}
P(e \mid z) & =\frac{2}{\sqrt{M} \log _{2} \sqrt{M}} \sum_{k=1}^{\log _{2} \sqrt{M}} \sum_{i=0}^{\left(1-2^{-k}\right) \sqrt{M}-1} w(i, k, M) \\
& \times\left\{\alpha_{p} p \mathrm{Q}\left(\sqrt{a(i, M) z^{2} \frac{\delta_{\mathrm{g}} \delta_{i}}{\delta_{\mathrm{g}}+\delta_{i}}}\right)\right. \\
& \left.+\left(1-\alpha_{p} p\right) \mathrm{Q}\left(\sqrt{a(i, M) z^{2} \delta_{\mathrm{g}}}\right)\right\}
\end{aligned}
$$

in which

$$
\begin{gathered}
w(i, k, M)=(-1)^{\left\lfloor\frac{i \cdot 2^{k-1}}{\sqrt{M}}\right\rfloor} \cdot\left(2^{k-1}-\left\lfloor\frac{i \cdot 2^{k-1}}{\sqrt{M}}+\frac{1}{2}\right\rfloor\right), \\
a(i, M)=\frac{3(2 i+1)^{2}}{(M-1)} \log _{2} M,
\end{gathered}
$$

$M$ is the order of the constellation, $\delta_{\mathrm{g}}$ is the signal-topermanent-noise ratio, defined as the ratio of the signal power to the power of the background Gaussian noise that is always present in the system, and $\delta_{i}$ is the signal-to-impulsive-noise ratio, defined as the ratio between the power of the signal and the power of the impulsive noise that acts in the system. 
Making

$$
x=z^{2}
$$

in $(2), P(e \mid z)$ can be written as

$$
\begin{aligned}
P(e \mid z=\sqrt{x}) & =\frac{2}{\sqrt{M} \log _{2} \sqrt{M}} \sum_{k=1}^{\log _{2} \sqrt{M}} \sum_{i=0}^{\left(1-2^{-k}\right) \sqrt{M}-1} w(i, k, M) \\
& \times\left\{\alpha_{p} p \mathrm{Q}\left(\sqrt{a(i, M) x \frac{\delta_{\mathrm{g}} \delta_{i}}{\delta_{\mathrm{g}}+\delta_{i}}}\right)\right. \\
& \left.+\left(1-\alpha_{p} p\right) \mathrm{Q}\left(\sqrt{a(i, M) x \delta_{\mathrm{g}}}\right)\right\} .
\end{aligned}
$$

The bit error probability, $P_{e}$, can be obtained by taking the average of (6) with respect to the PDF of $x$, that is,

$$
\begin{aligned}
P_{e} & =\int_{0}^{\infty} P(e \mid z=\sqrt{x}) f_{X}(x) \mathrm{d} x \\
& =\frac{2}{\sqrt{M} \log _{2} \sqrt{M}} \sum_{k=1}^{\log _{2} \sqrt{M}\left(1-2^{-k}\right) \sqrt{M}-1} \sum_{i=0}^{\infty} w(i, k, M) \\
& \times\left\{\alpha_{p} p \int_{0}^{\infty} \mathrm{Q}\left(\sqrt{a(i, M) x \frac{\delta_{\mathrm{g}} \delta_{i}}{\delta_{\mathrm{g}}+\delta_{i}}}\right) f_{X}(x) \mathrm{d} x\right. \\
& \left.+\left(1-\alpha_{p} p\right) \int_{0}^{\infty} \mathrm{Q}\left(\sqrt{a(i, M) x \delta_{\mathrm{g}}}\right) f_{X}(x) \mathrm{d} x\right\},
\end{aligned}
$$

in which $f_{X}(x)$ is the PDF of the fading.

An alternative expression for the function $\mathrm{Q}(\cdot)$ is given by $[15]$

$$
\mathrm{Q}(x)=\frac{1}{\pi} \int_{0}^{\frac{\pi}{2}} \exp \left(-\frac{x^{2}}{2 \sin ^{2} \theta}\right) \mathrm{d} \theta .
$$

Thus, $P_{e}$ can be written as

$$
\begin{aligned}
P_{e} & =\frac{2}{\pi \sqrt{M} \log _{2} \sqrt{M}} \sum_{k=1}^{\log _{2} \sqrt{M}} \sum_{i=0}^{\left(1-2^{-k}\right) \sqrt{M}-1} w(i, k, M) \\
& \times\left\{\alpha_{p} p \int_{0}^{\frac{\pi}{2}} \int_{0}^{\infty} \exp \left(-\frac{a(i, M) x}{2 \sin ^{2} \theta} \frac{\delta_{\mathrm{g}} \delta_{i}}{\delta_{\mathrm{g}}+\delta_{i}}\right) f_{X}(x) \mathrm{d} x \mathrm{~d} \theta\right. \\
& \left.+\left(1-\alpha_{p} p\right) \int_{0}^{\frac{\pi}{2}} \int_{0}^{\infty} \exp \left(-\frac{a(i, M) x}{2 \sin ^{2} \theta} \delta_{\mathrm{g}}\right) f_{X}(x) \mathrm{d} x \mathrm{~d} \theta\right\} .
\end{aligned}
$$

Since the fading is constant for at least one symbol interval $T_{\mathrm{s}}$, it is possible to represent the behavior of the channel by means of a Markov chain of $N$ states. In the model considered in this article, whenever the fading changes to a particular state, it is assumed that it will remain in this state for a time $T_{i}=k T_{\mathrm{s}}$ seconds. The parameter $T_{i}$ is a discrete random variable with distribution $p_{T_{i}}(t)$ and average value $\overline{t_{i}}$. In a given state $j$, the fading envelope is characterized by a random variable $X_{j}$ with probability density function $f_{X_{j}}(x)$. Under these conditions, the probability of $X \leq x$ can be written as

$$
\begin{aligned}
P(X \leq x) & =\sum_{i=1}^{N} \sum_{j=1}^{N} P\left(X \leq x, T_{i}, e_{j}\right) \\
& =\sum_{i=1}^{N} \sum_{j=1}^{N} P\left(X \leq x \mid T_{i}, e_{j}\right) P\left(T_{i}, e_{j}\right) .
\end{aligned}
$$

Given that [18]

$$
P\left(T_{i}, e_{j}\right)=\frac{P\left(e_{j}\right) \overline{t_{i}} \delta[i-j]}{\sum_{u=1}^{N} \sum_{v=1}^{N} \overline{t_{u}} P\left(e_{v}\right) \delta[u-v]},
$$

where $\delta[i-j]$ is the Kronecker delta, which assumes value equal to one if $i=j$ and zero otherwise; $\bar{t}_{i}$ is the average duration of the $i$-th state and $P\left(e_{j}\right)$ is the probability that the chain be in the $j$-th state after $n$ transitions, it follows that

$$
\begin{aligned}
P(X \leq x) & =\sum_{i=1}^{N} \sum_{j=1}^{N} P\left(X \leq x \mid T_{i}, e_{j}\right) \\
& \times \frac{P\left(e_{j}\right) \bar{t}_{i} \delta(i-j)}{\sum_{u=1}^{N} \sum_{v=1}^{N} \overline{t_{u}} P\left(e_{v}\right) \delta[u-v]} \\
& =\sum_{j=1}^{N} P\left(X \leq x \mid T_{j}, e_{j}\right) \frac{P\left(e_{j}\right) \overline{t_{j}}}{\sum_{v=1}^{N} \overline{t_{v}} P\left(e_{v}\right)} \\
& =\sum_{j=1}^{N} P\left(X_{j} \leq x\right) \frac{P\left(e_{j}\right) \overline{t_{j}}}{\sum_{v=1}^{N} \overline{t_{v}} P\left(e_{v}\right)} .
\end{aligned}
$$

Hence, the probability density function $f_{X}(x)$ is obtained from the derivative of the cumulative distribution function $F_{X}(x)=P(X \leq x)$ and can be written as

$$
f_{X}(x)=\sum_{j=1}^{N} f_{X_{j}}(x) \frac{P\left(e_{j}\right) \overline{t_{j}}}{\sum_{v=1}^{N} \overline{t_{v}} P\left(e_{v}\right)},
$$

in which $f_{X_{j}}(x)$ is the PDF of $X$ in the $j$-th state of the Markov chain.

A simplification of this model can be performed considering that the chain remains in a certain state during a symbol interval and then performs a transition to another state. This means that all states have the same average duration value, since fading can be considered independent of time for at least one symbol interval $T_{\mathrm{s}}$. Considering a Markov chain of $N$ states, the probability of $X \leq x$ can be written as

$$
\begin{aligned}
P(X \leq x) & =\sum_{j=1}^{N} P\left(X \leq x, e_{j}\right) \\
& =\sum_{j=1}^{N} P\left(X \leq x \mid e_{j}\right) P\left(e_{j}\right) \\
& =\sum_{j=1}^{N} P\left(X_{j} \leq x\right) P\left(e_{j}\right) .
\end{aligned}
$$


Hence, the $\operatorname{PDF} f_{X}(x)$ is given by

$$
f_{X}(x)=\sum_{j=1}^{N} f_{X_{j}}(x) P\left(e_{j}\right)
$$

in which $f_{X_{j}}(x)$ is the PDF of $X$ in the $j$-th state of the Markov chain and $P\left(e_{j}\right)$ is the probability that the chain be in the $j$-th state.

Substituting (15) in (9), it follows that

$$
\begin{aligned}
P_{e} & =\frac{2}{\pi \sqrt{M} \log _{2} \sqrt{M}} \sum_{k=1}^{\log _{2} \sqrt{M}} \sum_{i=0}^{\left(1-2^{-k}\right) \sqrt{M}-1} \sum_{j=1}^{N} w(i, k, M) \\
& \times P\left(e_{j}\right) \\
& \times\left\{\alpha_{p} p \int_{0}^{\frac{\pi}{2}} \int_{0}^{\infty} \exp \left(-\frac{a(i, M) x}{2 \sin ^{2} \theta} \frac{\delta_{\mathrm{g}} \delta_{i}}{\delta_{\mathrm{g}}+\delta_{i}}\right) f_{X_{j}}(x) \mathrm{d} x \mathrm{~d} \theta\right. \\
& \left.+\left(1-\alpha_{p} p\right) \int_{0}^{\frac{\pi}{2}} \int_{0}^{\infty} \exp \left(-\frac{a(i, M) x}{2 \sin ^{2} \theta} \delta_{\mathrm{g}}\right) f_{X_{j}}(x) \mathrm{d} x \mathrm{~d} \theta\right\} .
\end{aligned}
$$

Given that [19]

$$
\int_{0}^{\infty} e^{-s x} f_{X}(x) \mathrm{d} x=M_{X}(s)
$$

in which $M_{X}(s)$ represents the MGF of $f_{X}(x)$, it follows that it is possible to write (16) as

$$
\begin{aligned}
P_{e} & =\frac{2}{\pi \sqrt{M} \log _{2} \sqrt{M}} \sum_{k=1}^{\log _{2} \sqrt{M}} \sum_{i=0}^{\left(1-2^{-k}\right) \sqrt{M}-1} \sum_{j=1}^{N} w(i, k, M) \\
& \times P\left(e_{j}\right)\left\{\alpha_{p} p \int_{0}^{\frac{\pi}{2}} M_{X_{j}}\left(\frac{a(i, M)}{2 \sin ^{2} \theta} \frac{\delta_{\mathrm{g}} \delta_{i}}{\delta_{\mathrm{g}}+\delta_{i}}\right) \mathrm{d} \theta\right. \\
& \left.+\left(1-\alpha_{p} p\right) \int_{0}^{\frac{\pi}{2}} M_{X_{j}}\left(\frac{a(i, M) \delta_{\mathrm{g}}}{2 \sin ^{2} \theta}\right) \mathrm{d} \theta\right\} .
\end{aligned}
$$

For the model $\mathrm{G}^{2} \mathrm{AWGN}$, the bit error probability conditioned to $z$ is given by [17]

$$
\begin{aligned}
P(e \mid z) & =\frac{2}{\sqrt{M} \log _{2} \sqrt{M}} \sum_{k=1}^{\log _{2} \sqrt{M}} \sum_{i=0}^{\left(1-2^{-k}\right) \sqrt{M}-1} w(i, k, M) \\
& \times\left\{\alpha_{p} \beta p_{1} p_{2} \mathrm{Q}\left(\sqrt{a(i, M) z^{2} \frac{\delta_{\mathrm{g}} \delta_{i}}{\delta_{\mathrm{g}}+\delta_{i}}}\right)\right. \\
& \left.+\left(1-\alpha_{p} \beta p_{1} p_{2}\right) \mathrm{Q}\left(\sqrt{a(i, M) z^{2} \delta_{\mathrm{g}}}\right)\right\} .
\end{aligned}
$$

Using (5), it follows that $P(e \mid z=\sqrt{x})$ is given by

$$
\begin{aligned}
P(e \mid z=\sqrt{x}) & =\frac{2}{\sqrt{M} \log _{2} \sqrt{M}} \sum_{k=1}^{\log _{2} \sqrt{M}} \sum_{i=0}^{\left(1-2^{-k}\right) \sqrt{M}-1} w(i, k, M) \\
& \times\left\{\alpha_{p} \beta p_{1} p_{2} \mathrm{Q}\left(\sqrt{a(i, M) x \frac{\delta_{\mathrm{g}} \delta_{i}}{\delta_{\mathrm{g}}+\delta_{i}}}\right)\right. \\
& \left.+\left(1-\alpha_{p} \beta p_{1} p_{2}\right) \mathrm{Q}\left(\sqrt{a(i, M) x \delta_{\mathrm{g}}}\right)\right\}
\end{aligned}
$$

The average bit error probability for a Markov chain with $N$ states for the $\mathrm{G}^{2} \mathrm{AWGN}$ model is given by

$$
\begin{aligned}
P_{e} & =\frac{2}{\pi \sqrt{M} \log _{2} \sqrt{M}} \sum_{k=1}^{\log _{2} \sqrt{M}} \sum_{i=0}^{\left(1-2^{-k}\right) \sqrt{M}-1} \sum_{j=1}^{N} w(i, k, M) \\
& \times P\left(e_{j}\right)\left\{\alpha_{p} \beta p_{1} p_{2} \int_{0}^{\frac{\pi}{2}} M_{X_{j}}\left(\frac{a(i, M)}{2 \sin ^{2} \theta} \frac{\delta_{\mathrm{g}} \delta_{i}}{\delta_{\mathrm{g}}+\delta_{i}}\right) \mathrm{d} \theta\right. \\
& \left.+\left(1-\alpha_{p} \beta p_{1} p_{2}\right) \int_{0}^{\frac{\pi}{2}} M_{X_{j}}\left(\frac{a(i, M) \delta_{\mathrm{g}}}{2 \sin ^{2} \theta}\right) \mathrm{d} \theta\right\},
\end{aligned}
$$

in which $M_{X}(\cdot)$ represents the MGF.

\section{Moment Generating Function of the Distributions $\eta-\mu$ and $\kappa-\mu$}

If $z$ in (2) and (19) has distribution $\eta-\mu$ or $\kappa-\mu$, the probability density function of of $x=z^{2}$, in (6) and (20), is given by [19]

$$
f_{X}(x)=\frac{1}{2 \sqrt{x}}\left[f_{Z}(\sqrt{x})+f_{Z}(-\sqrt{x})\right]
$$
by

If $z$ has distribution $\eta$ - $\mu$, it follows that $f_{X}(x)$ is given

$$
\begin{aligned}
f_{X_{\eta-\mu}}(x) & =\frac{2 \sqrt{\pi} \mu^{\mu+0.5} h^{\mu} x^{\mu-0.5}}{\Gamma(\mu) H^{\mu-0.5} \Omega^{\mu+0.5}} \exp \left(-\frac{2 \mu h x}{\Omega}\right) \\
& \times \mathrm{I}_{\mu-0.5}\left(\frac{2 \mu H x}{\Omega}\right) \mathrm{u}(x)
\end{aligned}
$$

in which $\mathrm{u}(\cdot)$ represents the unit step function, $\Omega$ if the average power of the signal, $\Gamma(\cdot)$ represents the Gamma function and the parameters $H$ and $h$ are given by

$$
H=\frac{\eta^{-1}-\eta}{4} \quad \text { and } \quad h=\frac{2+\eta^{-1}+\eta}{4}
$$

for the first format and $\eta, 0<\eta<\infty$, is the ratio of the power of the phase and quadrature components. The generalized moment of that distribution is given by [20]

$$
M_{X_{\eta-\mu}}(s)=\left(\frac{4 \mu^{2} h}{(2(h-H) \mu+s \Omega)(2(h+H) \mu+s \Omega)}\right)^{\mu} .
$$


If $z$ has distribution $\kappa-\mu$, it follows that $f_{X}(x)$ is given by

$$
\begin{aligned}
f_{X_{\kappa-\mu}}(x) & =\frac{\mu(1+\kappa)^{\frac{\mu+1}{2}} x^{\frac{\mu-1}{2}}}{\kappa^{\frac{\mu-1}{2}} \exp (\kappa \mu) \Omega^{\frac{\mu+1}{2}}} \exp \left(-\frac{\mu(1+\kappa) x}{\Omega}\right) \\
& \times \mathrm{I}_{\mu-1}\left(2 \mu \sqrt{\frac{\kappa(1+\kappa) x}{\Omega}}\right) \mathrm{u}(x),
\end{aligned}
$$

in which the parameter $\kappa$ is the ratio of the total power of the dominant components and the power of the scattered waves, and $\Omega$ is the average power of the signal. The generalized moment for the distribution $\kappa-\mu$ is given by [20]

$$
M_{X_{\kappa-\mu}}(s)=\left(\frac{\mu(1+\kappa)}{\mu(1+\kappa)+s \Omega}\right)^{\mu} \exp \left(\frac{\mu^{2} \kappa(1+\kappa)}{\mu(1+\kappa)+s \Omega}-\mu \kappa\right) .
$$

\section{Results}

Simulations were carried out considering two channel scenarios. The first scenario is characterized by the occurrence of GAWGN noise and fading that can alternate between the models $\eta-\mu$ and $\kappa-\mu$, and the second scenario is characterized by the presence of noise $\mathrm{G}^{2}$ AWGN and fading $\eta-\mu$ or $\kappa-\mu$.

The values adopted for the Markov chain parameters are based on the work of Sanchez-Salas and Cuevas-Ruiz [8]. The values used for the parameters of the impulse noise $\eta(t)$ and the modulation schemes are chosen in accordance with impulsive interference studies described in [21] and with the conditions of simulations performed in [16].

In the present work, simulations were carried out by Monte Carlo method, considering $5 \times 10^{6}$ transmitted bits. In the simulations, the matrix of transition probabilities between states is given by [8]

$$
\mathbf{P}=\left[\begin{array}{ll}
0.7 & 0.3 \\
0.1 & 0.9
\end{array}\right]
$$

initially the states are equiprobable and the steady state is obtained with a high number of transitions between states The absence or presence of line of sight between transmitter and receiver is characterized by $\eta-\mu$ or $\kappa-\mu$ distribution, resp.

The average bit error probability curves of 64-QAM, for a Markov chain with two states, under the effects of noise GAWGN and $\eta-\mu$ or $\kappa-\mu$ fading are presented in Fig. 1 . The curves are plotted as a function of the signal-to-permanentnoise ratio, $\delta_{\mathrm{g}}$, for different values of $\delta_{i}$, with $p=0.3, \mu=2.0$, $\Omega=1.0, \kappa=2.0, \eta=0.3$, and $\alpha_{p}=0.1$. For low values of signal-to-impulsive-noise ratio, with $\delta_{i}=1 \mathrm{~dB}$ and $\delta_{i}=10 \mathrm{~dB}$, it is observed that the average bit error probability, $P_{e}$, changes little with the increase of $\delta_{\mathrm{g}}$, for values of signal-to-permanent-noise ratio in the range $20-40 \mathrm{~dB}$. For the four values of $\delta_{i}$ under consideration, it is observed, for $\delta_{\mathrm{g}}$ in the range 0 to $12 \mathrm{~dB}$, that the average bit error probability curves overlap. Figure 1 also presents the theoretical $P_{e}$ curve with the Markov chain subjected to AWGN and fading $\eta$ - $\mu$ or $\kappa-\mu$. It is observed that the curves with the presence of impulsive noise have a higher BEP value when compared to the theoretical curve of $P_{e}$ with the communication channel under the effect of AWGN. It is important to observe that the AWGN theoretical curve can be seen as a lower bound of the system performance.

It is also observed in Fig. 1 that the average BEP curves tend to become irreducible from a given signal-to-permanentnoise ratio. For low values $\delta_{i}$, such as $1 \mathrm{~dB}$, one has, for $\delta_{\mathrm{g}}=20 \mathrm{~dB}$, that the power of the impulsive noise is approximately 80 times greater than the power of the permanent noise, contributing so that the average BEP does not decrease from a given value of $\delta_{\mathrm{g}}$. For high values of signalto-impulsive-noise ratio, such as $20 \mathrm{~dB}$, both permanent and impulsive noise have low power. This explains why the respective average BEP curve presents a greater approximation (when compared to the curves referring to the low signal-toimpulsive-noise ratio) to the curve determined by the AWGN noise and $\eta$ - $\mu$ or $\kappa-\mu$ fading. However, even for this case of high $\delta_{i}$, the average BEP also tends to become irreducible from a given value of $\delta_{\mathrm{g}}$, because in this scenario the power of the permanent noise is much larger than the power of the impulsive noise. This contributes to the receiver's decisionmaking regions becoming more indefinite and, consequently, correct decoding of the transmitted bits is impaired.

In Fig. 2 average bit error probability curves are presented, for different values of the modulation order $M$, for the channel modeled by a Markov chain with two states, under the effects of noise GAWGN and $\eta$ - $\mu$ or $\kappa-\mu$ fading, considering $\mu=2.0, \alpha_{p}=0.3, p=0.4, \eta=0.2, \kappa=2.0, \Omega=1.0$ and $\delta_{i}=20 \mathrm{~dB}$. As the parameter $M$ increases, the greater is the number of symbols present in the constellation, and consequently the receiver is more likely to make an incorrect decision. Therefore, in this scenario, for fixed values of signal-to-permanent-noise ratio, it follows that greater average bit error probabilities are obtained. For $\delta_{\mathrm{g}}$ ranging from 30 to $40 \mathrm{~dB}$, average bit error probability less than $10^{-5}$ is obtained with $M=16$. An average bit error probability of $10^{-3}$ is achieved with $\delta_{\mathrm{g}} \approx 15.0 \mathrm{~dB}$ for $M=16$ and with $\delta_{\mathrm{g}} \approx 26.6 \mathrm{~dB}$ for $M=256$. It is also observed in Fig. 2 that the presence of impulsive noise causes the BEP curves deviate from the curve obtained when only AWGN noise is present in the system.

In Fig. 3 average bit error probability curves of 64-QAM are presented for a Markov chain with two states, under GAWGN and $\eta-\mu$ or $\kappa-\mu$ fading, with $\mu=2.0, \eta=0.3$, $\Omega=1.0, \alpha_{p}=0.1, p=0.2$ and $\delta_{i}=20 \mathrm{~dB}$. The $P_{e}$ curves are plotted as a function of the signal-to-permanent-noise ratio, $\delta_{\mathrm{g}}$, for different values of $\kappa$. One observes in Fig. 3 that greater average bit error probability is obtained as $\kappa$ increases. It is also observed that the BEP curves for the four considered $\kappa$ values tend to decrease faster when noise in the channel is the AWGN. In turn, around $\delta_{\mathrm{g}}=20 \mathrm{~dB}$, when the power of the impulsive noise is greater than the power of the permanent AWGN noise, the BEP curves tend to decrease 
slowly when compared to AWGN curves. It is observed that $P_{e}=10^{-3}$ is obtained with $\delta_{\mathrm{g}} \approx 17.3 \mathrm{~dB}$ for $\kappa=0.3$, while it is obtained with $\delta_{\mathrm{g}} \approx 21.1 \mathrm{~dB}$ for $\kappa=6.3$.

In Fig. 4, average bit error probability curves of 64-QAM are presented, for a Markov chain with two states, under the effects of noise $\mathrm{G}^{2} \mathrm{AWGN}$ and $\eta-\mu$ or $\kappa-\mu$ fading, with $\mu=2.0, \kappa=1.0, \Omega=1.0, \eta=1.5, \alpha_{p}=0.5$, $p_{2}=0.5, \beta=0.5$ and $p_{1}=0.5$, for four different values of signal-to-impulsive-noise ratio, $\delta_{i}$. For comparative purposes, the BEP curve is also shown in Fig. 4 when the Markov chain is subjected to AWGN and $\eta-\mu$ or $\kappa-\mu$ fading. It is observed that the BEP decreases as $\delta_{\mathrm{g}}$ increases, for fixed values of $\delta_{i}$. As the value of $\delta_{i}$ increases, it is noted that the BEP curves tend to follow the curve provided for the case where only AWGN noise and fading are present in the channel. In addition, it is observed that values of $P_{e}$ less than $10^{-4}$ are obtained only with high values of $\delta_{i}$, such as $20 \mathrm{~dB}$.

Average bit error probability curves for four values of modulation order $M$, for a channel modeled by a Markov chain with two states, subjected to noise $\mathrm{G}^{2}$ AWGN and $\eta$ - $\mu$ or $\kappa-\mu$ fading, are presented in Fig. 5, for $\delta_{i}=20 \mathrm{~dB}, \mu=2.0$, $\kappa=1.0, \Omega=1.0, \eta=1.5, \alpha_{p}=0.5, p_{2}=0.5, \beta=0.5$

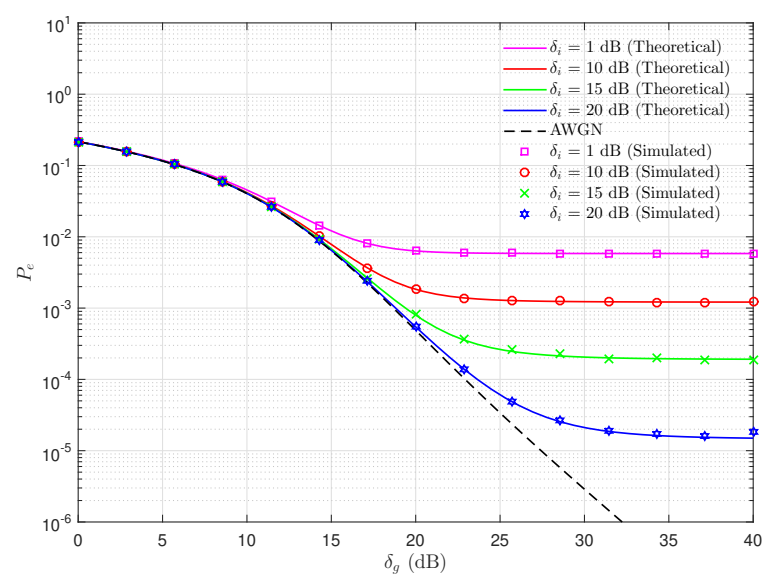

Fig. 1. Average BEP of 64-QAM, for a Markov chain with two states, under the effects of noise GAWGN and $\eta-\mu$ or $\kappa-\mu$ fading, for four different values of $\delta_{i}$.

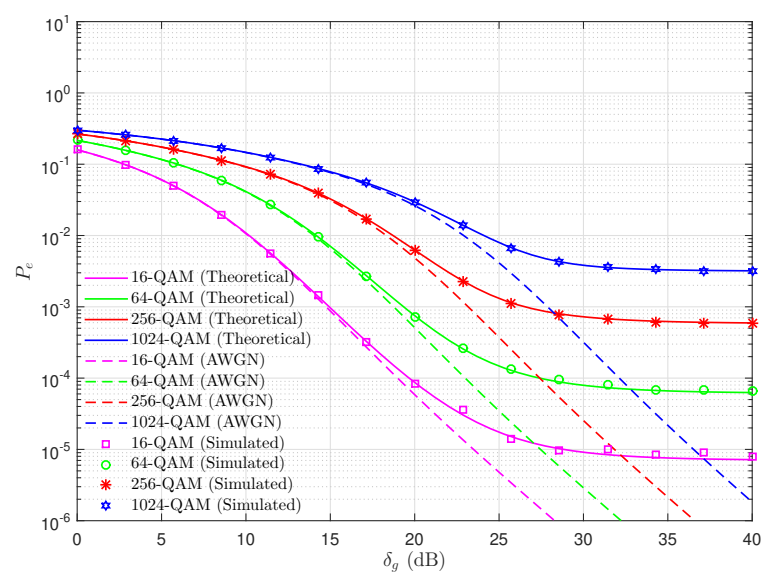

Fig. 2. Average BEP of $M$-QAM, for a Markov chain with two states, under the effect of noise GAWGN and $\eta-\mu$ or $\kappa-\mu$ fading, for different values of modulation order $M$ and $p_{1}=0.5$. The greater the number of symbols $M$ in the constellation, the closer the symbols affected by the noise and, consequently, the greater the average bit error probability for a fixed $\delta_{\mathrm{g}}$. An average bit error probability equal to $10^{-3}$ is obtained with $\delta_{\mathrm{g}} \approx 25.3 \mathrm{~dB}$ for $M=16$ and with $\delta_{\mathrm{g}} \approx 30.3 \mathrm{~dB}$ for $M=64$. For $\delta_{i}=20 \mathrm{~dB}$, it is observed that the average bit error probability, $P_{e}$, is not less than $10^{-3}$ for $\delta_{\mathrm{g}}<40 \mathrm{~dB}$, for constellations with $M \geq 64$.

In Fig. 6 average bit error probability curves of 64-QAM are presented for a Markov chain with two states, under the effects of noise $\mathrm{G}^{2} \mathrm{AWGN}$ and $\eta-\mu$ or $\kappa-\mu$ fading. The curves are plotted as a function of the signalto-permanent-noise ratio, $\delta_{\mathrm{g}}$, for different values of $\kappa$, with $\mu=2.0, \eta=1.5, \Omega=1.0, \alpha_{p}=0.5, p_{1}=0.5, p_{2}=0.5$ and $\delta_{i}=20 \mathrm{~dB}$. As $\kappa$ increases, a greater bit error probability is obtained. It is observed that an average bit error probability of $10^{-3}$ is obtained with $\delta_{\mathrm{g}} \approx 25.0 \mathrm{~dB}$ for $\kappa=0.3$, while it is obtained with $\delta_{\mathrm{g}} \approx 33.0 \mathrm{~dB}$ for $\kappa=6.3$. One observes in Fig. 6, by the dashed lines, that the $P_{e}$ curves of 64-QAM for a Markov chain with two states under the effects of AWGN and $\eta-\mu$ or $\kappa-\mu$ fading are practically linear for $\delta_{\mathrm{g}}$ in the range $20-40 \mathrm{~dB}$.

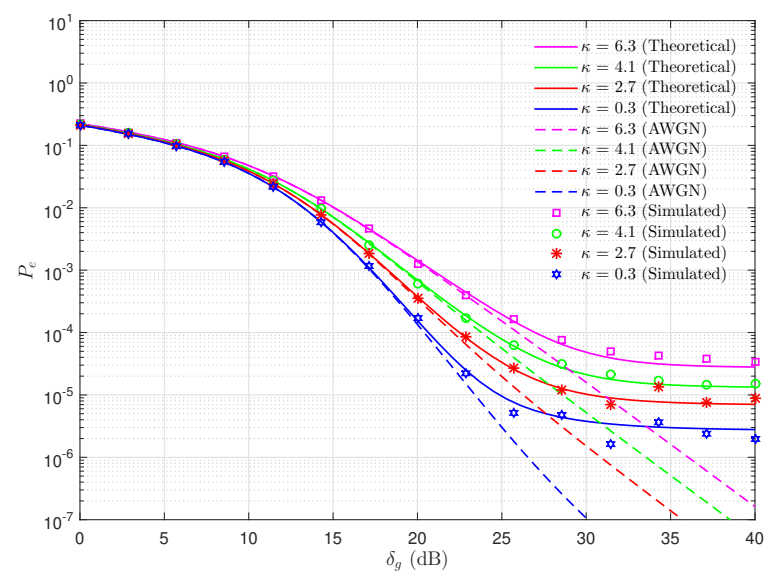

Fig. 3. Average BEP of 64-QAM for a Markov chain with two states, under the effect of noise GAWGN and $\eta-\mu$ or $\kappa-\mu$ fading, for different values of $\kappa$.

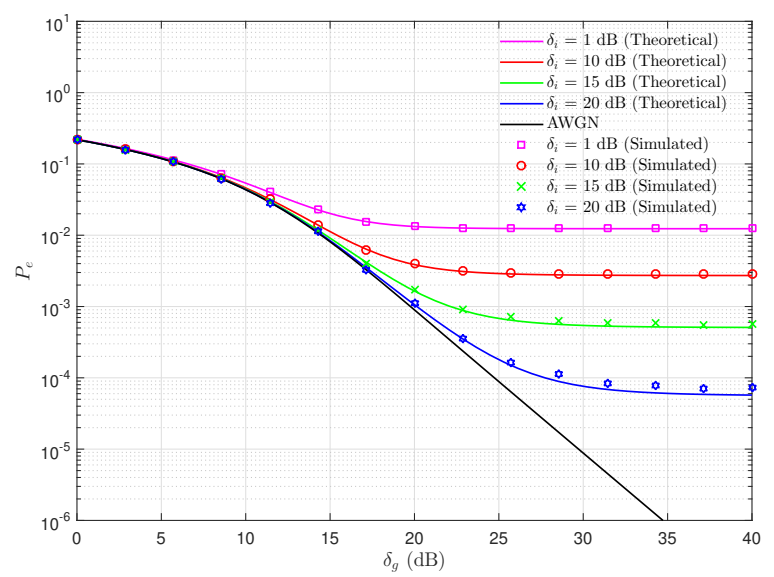

Fig. 4. Average BEP of 64-QAM, for a Markov chain with two states, under the effects of noise $\mathrm{G}^{2} \mathrm{AWGN}$ and $\eta-\mu$ or $\kappa-\mu$ fading, for different values of $\delta_{i}$. 


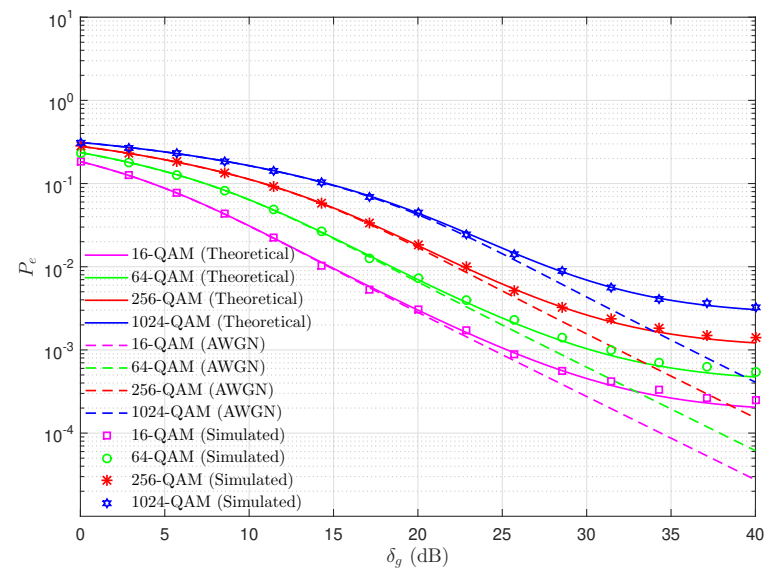

Fig. 5. Average BEP of $M$-QAM, for a Markov chain with two states, under the effects of noise $\mathrm{G}^{2} \mathrm{AWGN}$ and $\eta-\mu$ or $\kappa-\mu$ fading, for different values of modulation order $M$.

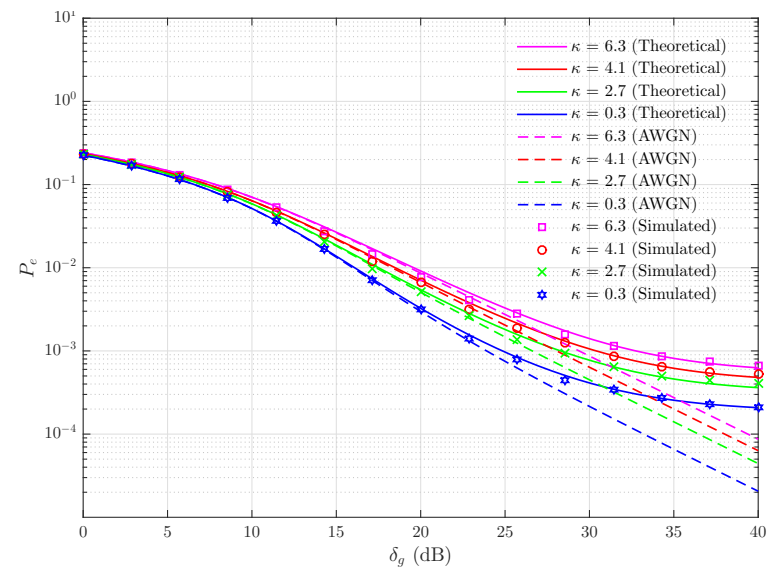

Fig. 6. Average BEP of 64-QAM for a Markov chain with two states, under the effects of noise $\mathrm{G}^{2} \mathrm{AWGN}$ and $\eta-\mu$ or $\kappa-\mu$ fading, for different values of $\kappa$.

\section{Conclusion}

This article presents new exact expressions for the average bit error probability, $P_{e}$, of the $M$-QAM scheme for a wireless communication channel model with fading driven by a Markov chain of $N$ states. For numerical evaluation of the mathematical expressions, a particular case of Markov chain with two states is considered, each one representing a different fading scenario. In the first state of the chain, the presence of the Gated Additive White Gaussian Noise (GAWGN) and fading $\eta-\mu$ or $\kappa-\mu$ is considered, whereas in the second state the presence of the Double Gated Additive White Gaussian Noise ( $\mathrm{G}^{2} \mathrm{AWGN}$ ) and fading $\eta-\mu$ or $\kappa-\mu$ is considered. Average bit error probability curves under different values of the signal-to-impulsive-noise ratio $\left(\delta_{i}\right)$, fading parameters and modulation order $M$ are shown.

In the simulation results, it has been observed that as the constellation order $M$ increases, the performance of the receiver is worse, and consequently larger values of $P_{e}$ are obtained for fixed values of the signal-to-permanent-noise ratio $\left(\delta_{\mathrm{g}}\right)$. Concerning the increase in the signal-to-impulsivenoise ratio, it was observed that some average bit error probability curves remained practically constant at certain intervals with the increase of $\delta_{\mathrm{g}}$, for $\delta_{i}$ equal to $1 \mathrm{~dB}$ and $10 \mathrm{~dB}$.

As future works, we aim to determine closed expressions for the average bit error probability of the modulation scheme $M$-QAM for a $N$ states Markov chain under the effects of GAWGN or $\mathrm{G}^{2} \mathrm{AWGN}$ and $\alpha-\mu$ fading [22].

\section{Acknowledgments}

This study was financed in part by the Coordenação de Aperfeiçoamento de Níve Superior - Brasil (CAPES) Finance Code 001 and the Conselho Nacional de Desenvolvimento Científico e Tecnológico (CNPq).

\section{References}

[1] TANGHE, E., JOSEPH, W., VERLOOCK, L., MARTENS, L., et al . The industrial indoor channel: Large-scale and temporal fading at 900, 2400, and $5200 \mathrm{MHz}$. IEEE Transactions on Wireless Communications, 2008, vol. 7, no. 7, p. 2740-2751. ISSN: 1536-1276. DOI: 10.1109/TWC.2008.070143.

[2] CHEFFENA, M. Propagation channel characteristics of industrial wireless sensor networks. IEEE Antennas and Propagation Magazine, 2016, vol. 58, no. 1, p. 66-73. ISSN: 1045-9243. DOI: 10.1109/MAP.2015.2501227

[3] TANG, L., WANG, K. C., HUANG, Y., GU, F. Channel characterization and link quality assessment of IEEE 802.15.4-compliant radio for factory environments. IEEE Transactions on Industrial Informatics, 2007, vol. 3, no. 2, p. 99-110. DOI: 10.1109/TII.2007.898414.

[4] HAYKIN, S. Communication Systems. 4th ed., John Wiley and Sons, 2002. ISBN: 9780471178699

[5] ZHANG, Q., KASSAM, S. Finite-state Markov model for Rayleigh fading channels. IEEE Transactions on Communications, 1999, vol. 47, no. 11, p. 1688-1692. ISSN: 0090-6778. DOI: $10.1109 / 26.803503$

[6] BABICH, F., LOMBARDI, G. A Markov model for the mobile propagation channel. IEEE Transactions on Vehicular Technology, 2000, vol. 49, no. 1, p. 63-73. DOI: 10.1109/25.820699

[7] PIMENTEL, C., FALK, T. H., LISBÔA, L. Finite-State Markov modeling of correlated Rician-fading channels. IEEE Transactions on Vehicular Technology, 2004, vol. 53, no. 5, p. 1491-1501. DOI: $10.1109 /$ TVT.2004.832413

[8] SANCHEZ-SALAS, D. A., CUEVAS-RUIZ, J. L. N-states channel model using Markov chains. In Proceedings of the Conference on Electronics, Robotics and Automotive Mechanics (CERMA). Cuernavaca (Mexico), 2007, p. 342-347. DOI: 10.1109/CERMA.2007.41

[9] OUYANG, W., ERYILMAZ, A., SHROFF, N. B. Downlink scheduling over Markovian fading channels. IEEE Transactions on Networking, 2016, vol. 24, no. 3, p. 1801-1812. DOI: 10.1109/TNET.2015.2438009. 
[10] LIU, X., LIU, C., LIU, W., ZENG, X. Wireless channel modeling and performance analysis based on Markov chain. In Proceedings of the Chinese Control and Decision Conference (CCDC). Chongqing (China), 2017, p. 2256-2260. ISSN: 1948-9447. DOI: $10.1109 /$ CCDC. 2017.7978890

[11] ALTINEL, D., KURT, G. K. Finite-state Markov channel based modeling of RF energy harvesting systems. IEEE Transactions on Vehicular Technology, 2018, vol. 67, no. 2, p. 1713-1725. DOI: 10.1109/TVT.2017.2757141

[12] LUTZ, E., CYGAN, D., DIPPOLD, M., et al. The land mobile satellite communication channel-recording, statistics, and channel model. IEEE Transactions on Vehicular Technology, 1991, vol. 40, no. 2, p. 375-386. DOI: 10.1109/25.289418

[13] VUCETIC, B., DU, J. Channel modeling and simulation in satellite mobile communication systems. IEEE Transactions on Vehicular Technology, 1992, vol. 10, no. 8, p. 1209-1218. ISSN: 0733-8716. DOI: $10.1109 / 49.166746$

[14] YACOUB, M. D. The $\eta-\mu$ and the $\kappa-\mu$ distribution. IEEE Antennas and Propagation Magazine, 2007, vol. 49, no. 1, p. 68-81. ISSN: 1045-9243. DOI: 10.1109/MAP.2007.370983

[15] CRAIG, J. W. A new, simple and exact result for calculating the probability of error for two-dimensional signal constellations. In Proceedings of the Military Communication Conference (MILCOM). McLean (USA), 1991, p. 571-575. DOI: 10.1109/MILCOM.1991.258319

[16] ARAÚJO, E. R., QUEIROZ, W. J. L., MADEIRO, F., et al. On gated Gaussian impulsive noise in M-QAM with optimum receivers. Journal of Communications and Information Systems, 2015, vol. 30, no. 1, p. 10-20. DOI: https://doi.org/10.14209/jcis.2015.2

[17] QUEIROZ, W. J. L., MADEIRO, F., LOPES, W. T. A., et al. On the performance of M-QAM for Nakagami channels subject to gated noise. Telecommunication Systems, 2018, vol 68, no. 1, p. 1-10. ISSN: 1572-9451. DOI: https://doi.org/10.1007/s11235-017-0371-7

[18] CHUNG, W., YAO, K. Modified hidden semi-Markov model for modelling the flat fading channel. IEEE Transactions on Communications, 2009, vol. 57, no. 6, p. 1806-1814. DOI: 10.1109/TCOMM.2009.06.070417

[19] LEON-GARCIA, A. Probability, Statistics, and Random Process for Electrical Engineering. 3rd ed., Pearson Prentice Hall, 2008. ISBN: 9780133002577

[20] ERMOLOVA, N. Y. Moment generating functions of the generalized $\eta-\mu$ and $\kappa-\mu$ distributions and their applications to performance evaluations of communication systems. IEEE Communications Letters, 2008, vol. 12, no. 7, p. 502-504. DOI: 10.1109/LCOMM.2008.080365.

[21] LAGO-FERNANDEZ, J., SALTER, J. Modelling Impulsive Interference in DVB-T: Statistical Analysis, Test Waveform \& Receiver Performance. 15 pages. [Online] Cited 2018-02-09. Available at: https://pdfs.semanticscholar.org/2a9d.pdf

[22] YACOUB, M. D. The $\alpha-\mu$ distribution: a physical fading model for the Stacy distribution. IEEE Transactions on Vehicular Technology, 2007, vol. 56, no. 1, p. 27-34. DOI: 10.1109/TVT.2006.883753

\section{About the Authors...}

Hugerles S. SILVA received his B.Sc. and M.Sc degrees in Electrical Engineering from Federal University of Campina Grande (UFCG), Brazil, in 2014 and 2016, respectively. His main research interests include optical communication systems, digital communication systems and coding theory.

Marcelo S. de ALENCAR received his Bachelor Degree in Electrical Engineering, from Federal University of Pernambuco (UFPE), Brazil, his Master Degree in Electrical Engineering, from Federal University of Paraíba (UFPB), Brazil, and his Ph.D. from the University of Waterloo, Canada. He worked for the Federal University of Paraiba (UFPB), for the Federal University of Campina Grande (UFCG), and for the State University of Santa Catarina (UDESC). Since 2017 he is with the Federal University of Bahia, Brazil. He is founder and President of the Institute for Advanced Studies in Communications (Iecom). He received achievement awards from the Brazilian Telecommunications Society (SBrT), from the Medicine College of the Federal University of Campina Grande (UFCG), and from the College of Engineering of the Federal University of Pernambuco. He published over 450 engineering and scientific papers and 22 books. He also wrote chapters for 11 books.

Wamberto J. L. de QUEIROZ received the B.Sc. degree in 1997 and the M.Sc. degree in 2002 from Federal University of Paraíba and the D.Sc. degree from Federal University of Campina Grande, Campina Grande, PB, in 2004, all in Electrical Engineering. From July 2004 to January 2005 he was with University Tiradentes, Aracaju, SE. From February 2005 to January 2007, he was with University of Fortaleza, CE, and from February 2007 to May 2010 he was with Federal University of Ceará. Since June 2010 he has been with the Department of Electrical Engineering of Federal University of Campina Grande. His research interests include signal spatial processing, diversity systems, channel modeling and estimation of parameters for wireless communication.

Rodrigo de A. COELHO received his B.Sc. degrees in Electrical Engineering from Federal University of Campina Grande (UFCG), Brazil, in 2015. His main research interests include power quality and power theory.

Francisco MADEIRO was born in Fortaleza, Ceará, Brazil, in 1972. He received his B.Sc., M.Sc and D.Sc. degrees in Electrical Engineering from Federal University of Paraíba (UFPB), Brazil, in 1995, 1998 and 2001, respectively. Since 2006 he is with University of Pernambuco (UPE), Brazil, where he is Associate Professor. His main research interests include signal processing, communications systems and computational intelligence. He was recipient of the Distinguished Award in Teaching at Polytechnic School of Pernambuco (POLI), UPE, in 2008 and 2013. He was recipient of the Distinguished Award in Research at POLI, in 2013. 